\title{
Physics of Transport Barriers
}

\author{
M. Tendler, G. VanOost*, L. Krlin ${ }^{+}$, R. Panek ${ }^{+}$, and J. Stockel ${ }^{+}$ \\ Fusion Plasma Physics, Alfvén Laboratory, Royal Institute of Technology, Stockholm, Sweden \\ * Department of Applied Physics, Ghent University, Belgium \\ ${ }^{+}$IPP Prague, Czech Republic
}

Received on 26 February, 2004

\begin{abstract}
The concept of ExB flow velocity shear suppression is utterly fundamental in modern fusion research. It is asserted that there are models enabling to understand the physics involved in LH transitions. To improve the understanding of the mechanisms leading to the formation of Transport Barriers, especially the relation between Internal and Edge barriers it is necessary to invoke the issue of electric fields. Edge transport barriers are the feature of the H-mode, the baseline regime of ITER, whereas Internal Transport Barriers are used to develop regimes that might be employed for steady state operation of ITER, definitely beneficial for design and operation of fusion power plants in the future. Their synergy will be addressed. Plasma flows are closely connected to electric fields. Therefore, their role is crucial for understanding of tokamaks aimed at the achievement of fusion energy. This appears in the well known neoclassical theory as the most accomplished and selfconsistent basis for understanding of fusion plasmas. It pertains to the novel concept of "zonal flows" emerging from the recent development of gyro-kinetic transport codes. The equilibrium poloidal and toroidal flows are also crucial for the concept of the electric field shear suppression of plasma turbulence in tokamaks. Yet, this timely and topical issue has remained largely unaddressed experimentally because of great difficulties in measuring flows in plasmas.
\end{abstract}

\section{Introduction}

The concept of ExB flow velocity shear suppression is utterly fundamental in modern fusion research. It is asserted that there are models enabling to understand the physics involved in LH transitions. To improve the understanding of the mechanisms leading to formation of Transport Barriers, especially the relation between Internal and Edge barriers it is necessary to invoke the issue of electric fields. Edge transport barriers are the feature of the $\mathrm{H}$-mode, the baseline regime of ITER-FEAT, whereas Internal Transport Barriers are used to develop regimes that might be employed for steady state operation of ITER -FEAT, definitely beneficial for design and operation of fusion power plants in the future.

Plasma flows are closely connected to electric fields. Therefore, their role is crucial for understanding of tokamaks aimed at the achievement of fusion energy. This appears in the well known neoclassical theory as the most accomplished and selfconsistent basis for understanding of fusion plasmas. It pertains to the novel concept of "zonal flows" emerging from the recent development of gyro-kinetic transport codes. The equilibrium poloidal and toroidal flows are also crucial for the concept of the electric field shear suppression of plasma turbulence in tokamaks. Yet, this timely and topical issue has remained largely unaddressed experimentally because of great difficulties of measuring flows in plasmas. These effects play an important role in the contemporary problems of transport in tokamaks. Problems emerge since the time scale for the suppression of turbulence cau- sed by the shear of the electric field if estimated from the conventional random walk arguments does not agree with measurements [1]. In addition, the ultimate necessity to reconcile models put forward to explain transitions into regimes with improved confinement with first principles should not be overlooked. To this end, it has been emphasised that models based only on ion-orbit loss mechanism do not satisfy this fundamental constraint due to the inherent inconsistency with the neoclassical theory [2]. The error emerges because the $\mathrm{j} x \mathrm{~B}$ force (caused either due to ion orbit losses or any other reason) has obviously no parallel component whereas the parallel neoclassical viscosity cancels exactly in the toroidal direction according to standard neoclassical theory [3].

Here, it is shown that many experimentally observed features may be assessed employing existing models. The bottom line is the sensitivity of the solution of transport equations with diffusivities governed by the shear of the electric field to subtle details of equilibrium pressure, density and temperature profiles. Indeed, they are dependent on both gradients and curvatures of evolving profiles [4,5]. Boundary conditions imposed on the interface of plasmas with discontinuous values of the electric field play also a crucial role yielding unconventional solutions. Hence, it is important to bear in mind that transport is not determined by local densities or temperatures, but by subtle details of their profiles thereby making diffusive time scales in principle irrelevant.

Indeed, the high sensitivity of a diffusivity (this may be 
particle, momentum, energy etc.) to subtle details of equilibrium profiles arising due to the dependence of fluxes on the second derivative of equilibrium profiles has been put to light within the context of the ExB shear suppression paradigm in $[4,5,14]$.

To this end, the notion of subthreshold transitions (lacking a bifurcation and below the power limit) has been brought about. Scenarios delivering this kind of transitions occur primarily due to the sensitivity to subtle details of plasma profiles. They have been coined "Tunneling Transitions" in analogy with quantum mechanics $[4,11]$.

Finally, an insight is offered addressing both Internal and Edge Transport Barriers (ITB \& ETB) within the context of the shear suppression concept. These might have their common origin caused by the interface of plasmas at a separatrix with $\mathrm{O}$ and $\mathrm{X}$ points arising due to different magnetic configurations of confinement in different regions of plasma bulk, thereby providing a large shear of the electric field. The simplest example of this idea takes place at the edge where the electric field within the SOL is governed by a contact with plates. This has to match the self-generated electric field on closed field lines governed by plasma flows and magnetic field geometry. Within the framework of neoclassical theory, the focus is on a confined region and this matching procedure may be provided by the impact of boundary conditions. Along the same lines, the ITB may stem from the necessity to force a constant electric field generated within a chain of magnetic islands (dwelling on rational q - surfaces) to match the neoclassical electric field primarily governed by temperature and pressure profiles. Therefore, the shear of the electric field emerges at rational q-surfaces naturally and selfconsistently. This may explain the emergence of the Internal Transport Barriers.

\section{The impact of the ExB shear and the origin of the electric field}

The bottom line of the E x B shear concept consists of two issues: the impact of the shear of an electric field on a residual turbulence and a more difficult question about a plausible origin of significant temporal and spatial variations of the electric field. Assuming an appearance of an optional electric field first, theory predicts effects of sheared E x B flow resulting from one of two mechanisms described in $[6,7]$. Complete stabilisation of various modes by the ExB rotational shear rate does occur provided

$$
\begin{gathered}
\alpha_{E}>\alpha \quad \max \\
\alpha_{E}=\frac{R B q}{B} \frac{\partial(E r / R B q)}{\partial r} ; \alpha_{\max } \text { linear growth rate }
\end{gathered}
$$

Therefore, the complete stabilisation is the mode specific feature providing for the ample suppression of a dominant turbulence channel (f.e. Ion Temperature Gradient mode driven turbulence in discharges with the ITB's). The main physical effect relies upon an amplified damping due to an interaction between an unstable mode with a nearby, stable mode and the resulting from it the increase of the Landau damping of an unstable mode.
However, a bulk of other modes usually unstable and detrimental to confinement under conventional tokamak operation mode such as Trapped Electron and/or Electron Temperature Gradient modes remains the cause of enhanced losses. Therefore, the concept of nonlinear suppression due to decorrelation of turbulent vortices has been introduced to describe the beneficial effect of the ExB shear on a residual turbulence. Indeed, the effective growth rate $\alpha_{c}$ can be shown to decrease dramatically according to

$$
\alpha_{c}=\left(\Delta \bar{\omega}_{D} \alpha_{E}^{2}\right)^{1 / 3}
$$

where the first term is the nonlinear decorrelation rate in absence of the E x B shear. In summary, both mechanisms have a potential to benefit confinement provided an option to affect the electric field may be found.

The second and more difficult issue is how plasma can generate an electric field profile capable to provide for stringent requirements imposed by Eqs. (1 and 2).

In order to elucidate on the origin of the electric field in plasma and possible causes for the omnipresence the following equation provides the first insight

$$
E_{r}=\frac{1}{Z_{i} e n_{i}} \frac{\partial p_{i}}{\partial r}-B_{\phi} v_{\theta}+B_{\theta} v_{\phi}
$$

Hence, the beneficial impact of the electric field seems to be assured if an electric field Er and contributing to it poloidal and toroidal rotation velocities provides for the stringent requirement imposed on the shear. Therefore, there are basically three "knobs" (diamagnetic drift, poloidal and toroidal rotations) affecting the radial electric field. In real conditions, all of them evolve both in space ant in time thereby contributing to the complexity of the issue.

According to standard neoclassical theory (i.e. ignoring regions with steep gradients of plasma parameters) this is found to yield [8]

$$
E_{r}^{(N E O)}=\frac{T_{i}}{e}\left[\frac{d \ln p}{d r}-k \frac{d \ln T_{i}}{d r}\right]
$$

where $k$ is the constant dependent on collisionality.

Although Eq.(4) is the subject to stringent constraints (the absence of unbalanced neutral beam injection and the lack of regions with steep gradients of the order of the poloidal gyroradius) it provides many useful insights, in particular during an initial stage of a plausible transition. Indeed, employing Eq.(4) the shear of the electric field results to depend on both gradients (first derivatives) and curvatures (second derivatives) of pressure and temperature profiles.

Employing the first term in square parenthesis for the selfconsistent electric field as yielded by the neoclassical theory one obtains an important estimate for the shear of the electric field

$$
d E r / d r \sim d^{2} \ln p / d r^{2} \sim\left[-\left(p^{\prime} / p\right)^{2}+p^{\prime \prime} / p\right]
$$

Focussing on the first term the beneficial effect for a transition of an amplification of the pressure gradient without changing the local pressure at the same time is clearly seen from Eq. (5) because it provides more shear for 
given values of other parameters.Furthermore, it is obvious that for a given gradient of a pressure profile, it is easier to trigger a transition scenario at locations with a lower pressure, f.e. at the edge of a confined region born out during the LH transition. Hence, it becomes evident why LH transitions were discovered first among the other regimes with improved confinement. Measurements on D III-D show that edge gradients are found to consistently increase in time in discharges which make the LH transitions [14]. Moreover, there is a well-defined boundary between $\mathrm{L}$ mode and the $\mathrm{H}$ mode states in the gradient operational state. This result appear to be consistent with the present model. Notwithstanding transparency and clarity of arguments based upon Eqs. (4 and 5), it is important to bear in mind that within a spatial range of a plasma body characterised by steep gradients (f.e. plasma parameters within the transport barrier) the neoclassical expression Eq. (4) cannot be adopted because assumptions employed in deriving this are not justified. Therefore, the electric field has been found selfconsistently from the poloidal rotation balance in [8]. Note that it can be shown that Eqs. (4 and 5) unfold when neoclassical terms dominate. In other words, the neoclassical parallel viscosity (in the American literature also called "magnetic pumping") is the governing factor. Yet, the crucial test in validating different models for LH transitions transpires through experiments using a biased electrode. These experiments allow an electric field to be imposed at the plasma edge in a tokamak in a controlled manner in contrast to the natural electric field yielded by Eq. (3). Hence, more information is obtained by introducing a new variable the current, which is zero otherwise due to ambipolarity, thereby increasing the dimensionality of the parameter space. This approach has demonstrated unfolding LH transitions on many limiter tokamaks, which are not available naturally, due to substantial current densities [8] in contrast to predictions of standard neoclassical theory [2]. Note that the latter yields the biasing current to be exactly zero if the anomalous momentum diffusivity is neglected. However, accounting for anomalous transport caused by a turbulence the relation between applied voltage and the radial current has been obtained in [8]. In conclusion, the biasing experiments offer a crucial test in verifying different theories for the poloidal and toroidal rotation and models for LH transitions.

sectionQuantitative features of the model

The transport barrier is modelled by diffusion equations with arbitrary diffusivities taken as functions of the shear of the electric field $\mathrm{dE} / / \mathrm{dr} \sim \alpha$.

Hence, the system of governing equations reads

$$
\frac{\partial p}{\partial t}-\frac{\partial}{\partial r}\left[D(\alpha) \frac{\partial p}{\partial r}-V(\alpha) p\right]=S
$$

This model equation is assumed to describe any kind of transport such as the particle transport, the heat transport, the toroidal and poloidal momentum transport and the turbulence transport.

The parameter $\boldsymbol{\alpha}$ is proportional to the shear of the radial electric field. Tentative plot of diffusivities as the function of shear is shown in Fig. 1.

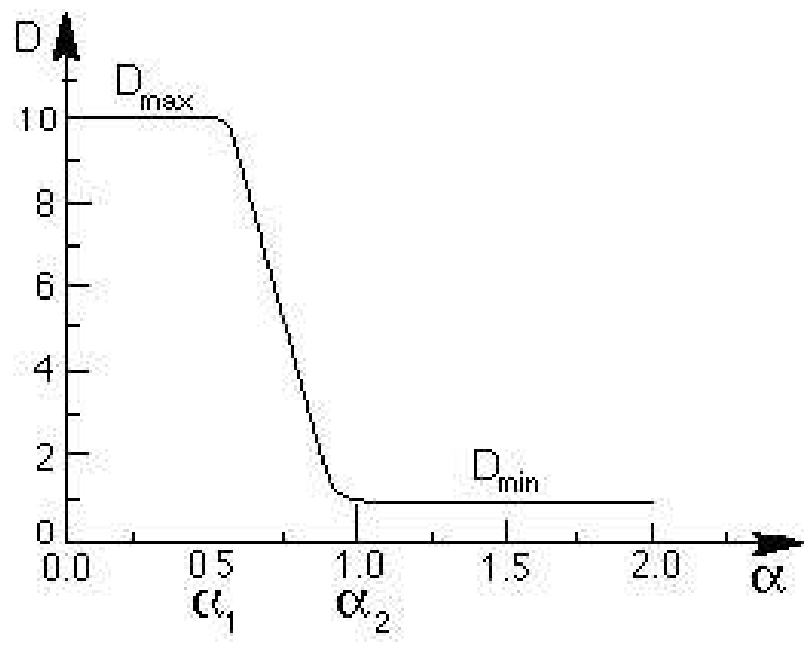

Figure 1. Diffusivities versus shear of the electric field.

At first, in order to gain insights into the physics governing transport under these assumptions few comments following from simple analytical considerations are offered. To this end, we wish to make an important point commenting on the crucial importance of the transport dependence on the first order versus the second order derivatives of plasma profiles. In more detail, the flux either emerges to be a function of only gradients and/or curvature of pressure and temperature profiles. If the former situation is the case then the plot of the flux $\Gamma=-D d p / d r$ versus the gradient takes the useful form yielding the effect of the bifurcation usually invoked in order to explain the $\mathrm{L} \mathrm{H}$ transition (the $\mathrm{S}$ - curve see Fig. 2 ) [9,10]. Therefore, ignoring the dependence of the shear of the electric field on the second derivative of a profile the scenario of the transition is significantly (over) simplified and emerges either as dwelling on the left or the right branches of this curve. The straightforward and fast transition occurs then $\alpha>\alpha$ max. The case of $\alpha=\alpha$ max describes the situation when prelude profiles prior to the transition represent an equilibrium unstable to an arbitrary perturbation. Therefore, a scenario of the transition caused entirely due to the diamagnetic drift (without employing a change in plasma rotation see Eq. 4) emerges.

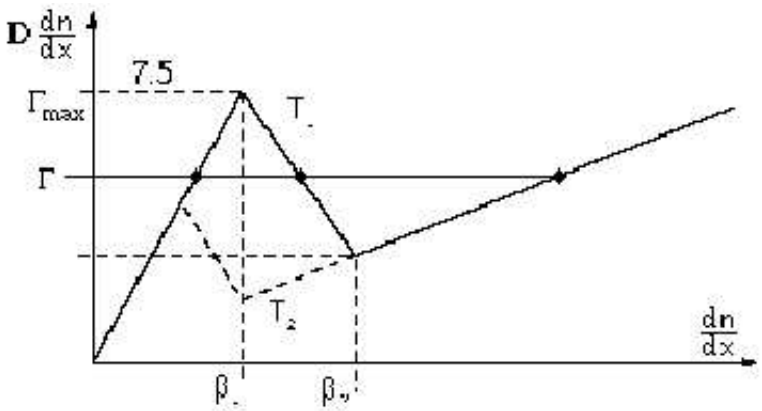

Figure 2. Fluxes dependence on gradients neglecting second derivatives.

Importantly, the explicit dependence of the shear on the curvature merits a special emphasis because this enables a transition below the threshold $\alpha<\alpha$ max. This result is 
supported both by analytical and numerical calculations [4]. This scenario has been coined "tunneling" transitions in analogy with quantum mechanics. This may be brought about at the plasma edge by injecting small pellets tangentially as shown also in recent experiments addressing $\mathrm{H}$-mode and Internal Transport Barriers carried out on the DIII-D tokamak [11], where H-mode plasmas have been directly produced by injecting frozen deuterium pellets into L-mode plasmas. The pellet triggers the $\mathrm{H}$-mode transition at lowered edge temperatures indicating that a critical edge temperature is not required for the H-mode transition. The pellets are also able to lower the threshold power required to produce the $\mathrm{H}$-mode transition indicating an agreement with predictions of the model.

\section{The Causality of Transitions}

At this point, the issue of causality for transitions into regimes with improved confinement such as the $\mathrm{L} \mathrm{H}$ transition comes to mind. Indeed, the problem is whether the trigger is due to a change in the rotational $\mathrm{Vx} \mathrm{B}$ term or the pressure gradient term of main ions according to Eq.(3). Here, it is important to keep in mind that a steep pressure gradient has a potential to provide for large local values of the electric field by its own virtue. However, this effect can be either counteracted or reinforced depending on a quantitative impact rendered by both poloidal and toroidal rotations. In more detail, the scenario of the transition resulting from solutions of the model Eq.(6) yields a very fast propagation of the front of improved confinement (see Fig. 3) in comparison with the random walk estimate (straight line in Fig. 3) based upon the conventional diffusive model. The main reason for this is a fast transport of the decorrelation of fluctuations carried up and down between the moving front of improved confinement and a separatrix. Therefore, the solution of the model Eq. (7) obtains "ballistic" features significantly reducing the effective time scale required by the transition. Hence, experimental studies showing the quench of turbulence on a very rapid time scale of the about $0.1 \mathrm{~ms}$ during the transition with bifurcation find its theoretical explanation.

In general, any effective time scale does not exist at all under these circumstances because of fundamental peculiarities of this kind of transport. Hence, the important signature of self organised systems relying upon intermittence and yielding avalanches is brought to light. Moreover, scenarios of a transition may be triggered very robustly by an infinitely small perturbation of the diamagnetic drift and/or rotation thereby making the issue of causality irrelevant. Indeed, they all merge very fast even under varying initial conditions [4].

Hence, the phenomenon of the transition is very robust and emerges under the right conditions. To this end, it has to be kept in mind that due to technical difficulties, it is almost impossible to answer questions related to causality experimentally because the resolution of the majority of diagnostics adopted on many devices is too slow to address the causal dependence experimentally. Furthermore, data reported from JET on the magnitude and sign of the poloidal rotation contradicts the same data obtained by the D III - D team.
Yet, a scenario of the transition triggered entirely by the diamagnetic term appears possible provided the given form of prelude profiles.

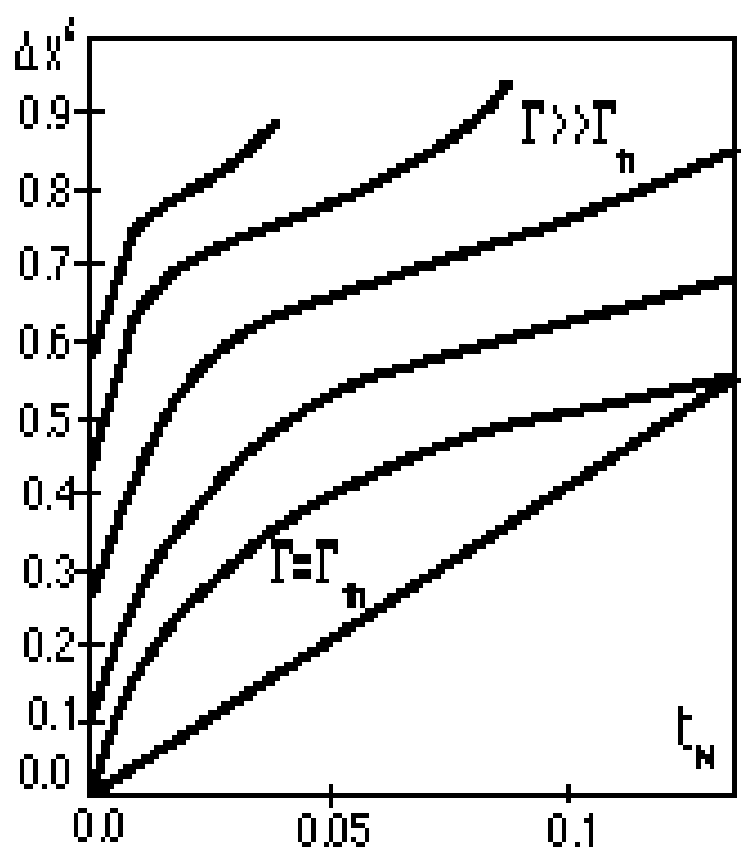

Figure 3. Propagation of the front of improved confinement.

Two different scenarios along these lines were demonstrated in [12]. It has been shown that the direct LH transition emerges provided the particle influx at the interface between the core and the thermal barrier was kept constant during the transition above the threshold value. In contrast when a possibility of the feedback has been taken into account the second (time dependent nonlinear regime) arises due to an interaction between inlet and outlet boundary conditions resulting from the feedback of the flux in the core on the flux into the SOL. The second constraint required obtaining a monotonic and time dependent solution is due to the dependence of the core flux on the varying density at the interface.

In the first scenario, the steepening of the density evolves gradually from the separatrix up the interface due to the reduced diffusion, thereby bringing about the bifurcation evolving into the thermal barrier. In the second scenario, the outflow at the separatrix $(\approx D \alpha$ signal $)$ dithers, being linked to the flux at the interface whereas absolute values of density vary insignificantly.

In general, if the dependence on the second derivative is taken into account the picture of the transition gets dramatically modified. Indeed, there is a more global relation strongly dependent on the distribution of sources of moments within the plasma.

Furthermore, even for a given distribution of sources, the relation between fluxes and gradients is not unique anymore (Fig. 2 is significantly modified). Hence, additional boundary conditions are required because the governing system of transport equations obtains the form of the higher order. 
Therefore, the peripheral plasma plays the crucial role in controlling the global confinement and affecting plasma profiles and local transport diffusivities. Moreover, properties of the plasma within the SOL must be within the restricted range if a stationary solution of transport equations is to be found at all.

Indeed, it has been shown before that a steady state solution does not emerge outside the narrow range of boundary conditions imposed on the pressure gradient at the separatrix. The only solution found for mixed Dirichlet boundary conditions describes autonomous oscillations implying dithering between the Low and the High Confinement. This regime has been also demonstrated on many devices. In summary, time dependent solutions appear to be a subtle phenomenon arising due to specific features of the model equation and the choice of boundary conditions. Recently, the data emerged indicating that the ITB are formed at rational flux surfaces with low q-values. Since the magnetic islands emerge in their vicinity, it is natural to assume the change of the electric field to cause the transition. In [13], the current caused by fast ions due to a fishbone activity is pointed to be a trigger for ITB's. However, the electric field is bound to change significantly by a chain of islands even without additional currents. Indeed, the electric field within the island remains constant or zero while outside this has to match the neoclassical value. The profile obtains a "stairs" like form with steps being determined by the scale depending on collisionality, toroidal rotation and anomalous viscosity [14]. In more detail, outside the island the radial electric field changes from the value determined by the island rotation towards the neoclassical value on the scale $\delta$, which depends on the collisionality and anomalous viscosity.

\section{Experimental results}

The impact of sheared radial electric fields on turbulent structures and flows at the plasma edge is investigated on the CASTOR tokamak $(\mathrm{R}=0.4 \mathrm{~m}, \mathrm{a}=0.085 \mathrm{~m})$. A non-intrusive biasing scheme called "eparatrix biasing" whereby the electrode is located in the scrape-off layer (SOL) with its tip just touching the LCFS was found to be efficient, as predicted by theory [8]. There is evidence of a strongly sheared radial electric field and ExB flow, resulting in the formation of a transport barrier at the separatrix. Advanced probe diagnosis of the edge region has shown that the EXB shear rate that arises during separatrix biasing is larger than for standard edge plasma biasing (with the electrode inserted inside the LCFS as in TEXTOR). The plasma flows, especially the poloidal ExB drift velocity, are strongly modified in the sheared region, reaching Mach numbers as high as half the sound speed. The corresponding shear rates $\left(\sim 5 \times 10^{6} \mathrm{~s}^{-1}\right)$ derived from both the flow and electric field profiles are in excellent agreement and are at least an order of magnitude higher than the growth rate of unstable turbulent modes as estimated from fluctuation measurements.

The optimized Gundestrup probe was used to compare ion flows with the phase velocity of fluctuations moving poloidally across the Gundestrup collectors. The ion mass flow is measured by the standard arrangement, i.e. signals of all the segments are digitized at a standard sampling rate. From these data, the perpendicular Mach number of the ion flow is derived. Simultaneously, the signals from the most upstream and downstream pairs of the segments are recorded faster, at a sampling rate of $5 \mathrm{MS} / \mathrm{s}$. Then, the cross-correlation function is calculated and the transit time of a poloidally localized structure across the corresponding segments is deduced from the shift of its maximum. In Fig. 4 we compare four measurements of the poloidal velocity deduced from (1) the IGP polar diagrams, (2) the phase velocity of structures moving across the upstream segments, 3 ) the phase velocity of structures moving across the downstream segments, and (4) the ExB drift speed calculated from the radial electric field using the rake probe.
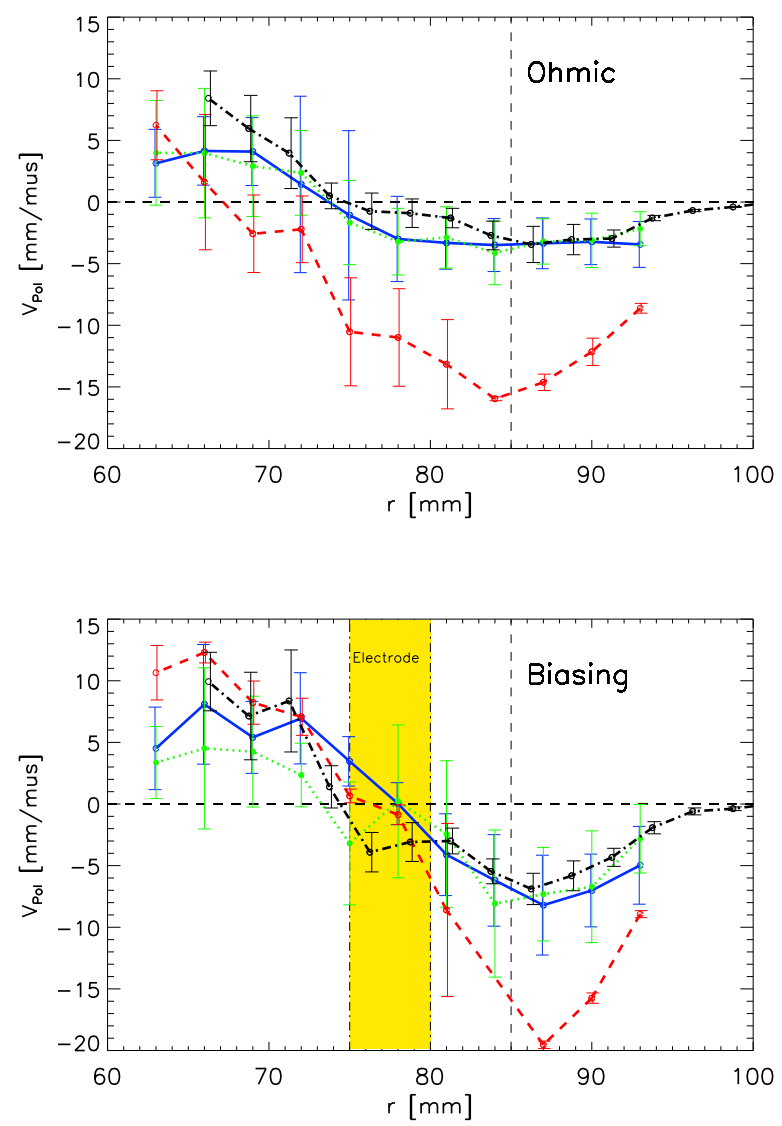

Figure 4. Radial profile of fluctuation velocities (solid and dotted), and flow velocity (dashed line) measured by the IGP. The black line is the ExB velocity deduced from the rake probe data. Left: ohmic phase of the discharge. Right: Biased phase of the discharge.

In both the ohmic and biased phases of the discharge, all methods give the same sign of the velocity and roughly the same inversion radius. From Fig. 4 it is evident that the ExB velocity and the fluctuation velocity follow each other very closely. In addition, the poloidal flow velocity is of the same order as the latter one within the separatrix $(r<75$ $\mathrm{mm}$ ). This indicates that turbulent structures are dragged by the poloidally rotating plasma. In contrast, there is a significant difference between the flow and ExB velocities observed within the SOL. The full explanation for this difference 
would require a better knowledge of the ion sound speed, which is needed to convert the Mach number, measured by the IGP to absolute units, $\mathrm{V}=\sqrt{ }\left[\left(\mathrm{T}_{e}+\mathrm{T}_{i}\right) / \mathrm{M}_{i}\right]$. The electron and ion temperatures are assumed to be equal, $\mathrm{T}_{e}=\mathrm{Ti}$ . However, our experiments indicate that the $\mathrm{T}_{e}$, deduced from the IV characteristics of the Langmuir probe can be overestimated by a factor of 2-3. Taking this into account, the difference between the flow and ExB velocities becomes smaller. Better agreement can be achieved, if the diamagnetic drift is taken into account, although in the case of CASTOR, the density and temperature profiles in the edge plasma are rather flat. The recent experimental data seem to confirm this conjectures.

Measurements of the parallel Mach numbers have also been performed at biasing. In contrast to Alcator C-Mod results at $\mathrm{L}-\mathrm{H}$ transition, we do not observe a dramatic increase of the toroidal rotation. This may be due to the much higher q values employed on CASTOR.

\section{Conclusions}

The model invoking the paradigm of shear suppression yields many effects observed experimentally. Even under the assumption of the neoclassical electric field, it yields novel insights and conclusions consistent with measurements. For example, it points us toward the insight that both inlet and outlet of the transport barrier might play a crucial role in determining the dynamics of the $\mathrm{L}-\mathrm{H}$ transitions in real tokamaks.

More importantly for applications, the concept of the "tunneling transition" has been put forward in analogy with the concept well-known from quantum mechanics relying upon the transition scenario emerging beyond the threshold and originating from the dependence on the second derivative. In general, it has to be kept in mind that the phenomena of transport barriers are very robust and sensitive only to prelude profiles. The most important practical conclusion from ideas and insights offered above is that the conventi- onal scenario of triggering the transition by means of the power exceeding a given threshold appears to be the "brute force" solution to obtain the ignition. More subtle and flexible methods appear to be underway.

\section{References}

[1] J. Hugill, Plasma Physics Controll. Fusion 42, R 75 (2000).

[2] A. A. Galeev and R. Sagdeev, Reviews of Plasma Physics vol.7, ed. M.A. Leontovich, Consultans Bureau, New York and London, 1979.

[3] Y.-B. Kim and F.L. Hinton, Phys. Fluids B4, 278 (1992).

[4] M. Tendler, Plasma Phys. Controll. Fusion B39, 371 (1997).

[5] J.B. Taylor, J.W. Connor, and P. Helander, Phys. Plasmas 5, 3065 (1998).

[6] H. Biglari, P. Diamond, and P.W. Terry, Phys. Fluids B 2, 1 (1990).

[7] T.S. Hahm and K. Burrel, Phys. Plasmas 2, 1648 (1995).

[8] V. Rozhansky and M. Tendler, Reviews of Plasma Physics vol.19, ed. B.B. Kadomtsev (New York and London, Consultants Bureau) (1996).

[9] F.L. Hinton Phys. Fluids B 3, 696 (1991).

[10] G.M. Staebler, R.E. Walz, and J.C. Wiley, Nuc. Fus. 37, 287 (1997).

[11] P. Gohil, Phys. Rev. Lett. 86, 644 (2001).

[12] V. Rozhansky, M. Tendler, and S. Voskoboinikov, Plasma Phys. Controll. Fusion 38, 1327 (1996)

[13] S. Guenter, Proc. 28 th EPS conf. on Plasma Physics and Controll. Fusion A 25, (ECA) p.1006.

[14] R. Groebner, D.M. Thomas, and R.D. Deranian ,Phys. Plasmas 8, 2722 (2001)

[15] E. Kaveeva and V.R. Rozhansky, Proc. 29 th EPS conf. on Plasma Physics and Controll. Fusion in print 